\title{
Vigilância ativa de eventos adversos pós- vacinação na atenção primária à saúde
}

Active surveillance of adverse events following immunization in primary health care Observación activa de eventos adversos posvacunación en la Atención Primaria de Salud

Emily Caroline Cardoso Batista' 1 in https://orcid.org//0000-0001-9108-2715

Ana Paula Ferreira ${ }^{1}$ (i) https://orcid.org/0000-0001-6933-1578

Valéria Conceição de Oliveira' ${ }^{1}$ ic https:/orcid.org/0000-0003-2006-9754

Gabriela Gonçalves Amaral' ${ }^{2}$ io https://orcid.org/0000-0002-9629-2815

Renato Fernando de Jesus ${ }^{1}$ io https://orcid.org/0000-0001-8344-0666

Nayara Dornela Quintino $0^{3}$ io https:/orcid.org/0000-0001-5740-0310

Selma Maria da Fonseca Viegas ${ }^{1}$ io https://orcid.org/0000-0002-0287-4997

Eliete Albano de Azevedo Guimarães ${ }^{1}$ ib hitps://orcid.org/0000-0001-9236-8643

Como citar:

Batista EC, Ferreira AP, Oliveira VC, Amaral GG, Jesus RF, Quintino ND, et al. Vigilância ativa de eventos adversos pós-vacinação na atenção primária à saúde. Acta Paul Enferm. 2021;34:eAPE002335.

DOI

http://dx.doi.org/10.37689/actaape/2021A0002335

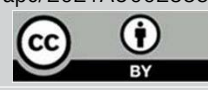

Descritores

Vacinação; Efeitos colaterais e reações adversas relacionados a medicamentos; Atenção primária à saúde; Monitoramento epidemiológico

Keywords

Vaccination; Drug-related side effects and adverse reactions; Primary health care; Epidemiological

monitoring

Descriptores

Vacunación; Efectos colaterales y reacciones adversas relacionados con medicamentos; atención primaria de salud; monitoreo epidemiológico

Submetido 25 de Agosto de 2020

Aceito 8 de Março de 2021

Autor correspondente Eliete Albano de Azevedo Guimarães E-mail: elietealbano@ufsj.edu.br

\section{Resumo}

Objetivo: Descrever a vigilância ativa dos eventos adversos pós-vacinação, sua incidência e fatores associados, em um município de Minas Gerais, Brasil.

Métodos: Coorte prospectiva realizada na Atenção Primária à Saúde, entre 2017 e 2018. Foram acompanhados 384 indivíduos que receberam vacinas, excluindo-se aqueles que tiveram eventos adversos prévios. Na linha de base, foram coletadas informações sociodemográficas, de saúde e histórico de vacinação e, no seguimento, as características do evento adverso e das ações de vigilância epidemiológica. Estimou-se taxa de incidência de eventos adversos, e realizaram-se 0 teste do qui-quadrado, a regressão de Poisson e 0 teste de Hosmer-Lemeshow.

Resultados: A incidência de eventos adversos foi de 13,36 casos/100 mil doses de vacinas (intervalo de confiança de 95\%: 13,34-13,38), com maior incidência em crianças menores de 5 anos. Os eventos adversos mais frequentes foram dor local, vermelhidão, endurecimento, seguidos de febre e choro persistente. Dentre os fatores associados à ocorrência dos eventos adversos, recebimento da vacina contra tétano e difteria (risco relativo: 7,9; intervalo de confiança de 95\%: 2,77-12,46) e administração por meio da via intramuscular foram considerados de risco (risco relativo: 6,1; intervalo de confiança de 95\%: 2,55-14,63). A conduta do profissional de enfermagem, diante das orientações sobre as vacinas recebidas, aumentou a notificação de eventos adversos (risco relativo: 3,4; intervalo de confiança de 95\%: 1,53-7,68).

Conclusão: 0 estudo permitiu conhecer fatores que favorecem a ocorrência de eventos adversos. Há evidências de que condutas adotadas pelos profissionais de enfermagem nas salas de vacinação podem evitar subnotificações de eventos adversos pós-vacinação.

\section{Abstract}

Objective: To describe the active surveillance of adverse events following immunization, their incidence and associated factors in a municipality of Minas Gerais, Brazil.

Methods: This is a prospective cohort conducted in Primary Health Care between 2017 and 2018. A total of 384 individuals who received vaccines were followed up, excluding those who had previous adverse events. At baseline, sociodemographic, health and vaccination history information and, in follow-up, the characteristics of adverse events and epidemiological surveillance actions were collected. The incidence rate of adverse events was estimated, and the chi-square test, poisson regression and Hosmer-Lemeshow test were performed.

Results: The incidence of adverse events was 13.36 cases $/ 100,000$ doses of vaccines $(95 \%$ confidence interval: 13.34-13.38), with a higher incidence in children under 5 years of age. The most frequent adverse events were local pain, redness, hardening, followed by fever and persistent crying. Among the factors

'Universidade Federal de São João del-Rei, Divinópolis, MG, Brasil.

${ }^{2}$ Escola de Enfermagem de Ribeirão Preto, Universidade de São Paulo, Ribeirão Preto, SP, Brasil.

${ }^{3}$ Secretaria de Estado de Saúde de Minas Gerais, Belo Horizonte, MG, Brasil.

Conflitos de interesse: nada a declarar. 
associated with the occurrence of adverse events, receiving tetanus and diffrhyphria vaccine (relative risk: 7.9; 95\% confidence interval: 2.77-12.46) and intramuscular administration were considered at risk (relative risk: $6.1 ; 95 \%$ confidence interval: 2.55-14.63). Nursing professionals' conduct, considering the guidelines on the vaccines received, increased adverse event reporting (relative risk: 3.4; 95\% confidence interval: 1.53-7.68).

Conclusion: The study allowed to know factors that favor the occurrence of adverse events. There is evidence that conducts adopted by nursing professionals in immunization rooms may avoid underreporting of adverse events following immunization.

\section{Resumen}

Observación: Describir la observación activa de los eventos adversos posvacunación, su incidencia y factores asociados en un municipio del estado de Minas Gerais, Brasil.

Métodos: Cohorte prospectiva realizada en la Atención Primaria de Salud, entre 2017 y 2018. Se realizó el seguimiento de 384 individuos que recibieron vacunas, excluyendo a aquellos que tuvieron eventos adversos previos. En la línea basal, se recopiló información sociodemográfica, de salud e historial de vacunación y, en el seguimiento, las características del evento adverso y las acciones de observación epidemiológica. Se estimó un índice de incidencia de eventos adversos y se realizó la prueba $\chi^{2}$ de Pearson, la regresión de Poisson y la prueba de Hosmer-Lemeshow.

Resultados: La incidencia de eventos adversos fue de 13,36 casos/100.000 dosis de vacuna (intervalo de confianza de 95 \%: 13,34-13,38), con mayor incidencia en niños menores de 5 años. Los eventos adversos más frecuentes fueron dolor local, enrojecimiento, endurecimiento, seguidos de fiebre y llanto persistente. Entre los factores asociados a la ocurrencia de los eventos adversos, la aplicación de la vacuna contra el tétanos y la difteria (riesgo relativo: 7,9; intervalo de confianza de $95 \%$ : 2,77-12,46) y la administración por medio de la vía intramuscular fueron considerados de riesgo (riesgo relativo: 6,1; intervalo de confianza de 95 \%: 2,55-14,63). La conducta del profesional de enfermería ante las instrucciones sobre las vacunas recibidas aumentó la notificación de eventos adversos (riesgo relativo: 3,4; intervalo de confianza de $95 \%$ : 1,53-7,68).

Conclusión: El estudio permitió conocer factores que favorecen a la ocurrencia de eventos adversos. Hay evidencias de que las conductas adoptadas por los profesionales de enfermería en las salas de vacunación pueden evitar subnotificaciones de eventos adversos posvacunación.

\section{Introdução}

Os imunobiológicos têm excelente registro de segurança, mas não estáo isentos de causar eventos adversos pós-vacinação, mesmo sendo submetidos a rigoroso processo de ensaio clínico, nos quais são exaustivamente testados e estudados, para garantir a máxima segurança e eficácia para seus usuários. ${ }^{(1)}$

Um evento adverso pós-vacinação é caracterizado como qualquer evento indesejável e não intencional que um indivíduo possa desenvolver ao receber uma dose de algum imunobiológico. ${ }^{(2)}$ Sua ocorrência pode estar relacionada ao processo de produçáo e armazenamento desses produtos, às características físicas e biológicas do indivíduo vacinado e ao processo de administraçáo desses imunobiológicos. ${ }^{(3,4)}$ Os eventos adversos pós-vacinação são classificados em dois tipos distintos: evento adverso grave e evento adverso não grave. Eles diferem em suas intensidades e gravidades, além do tipo de demanda por tratamento clínico. . $^{(3,5,6)}$

A ocorrência de evento adverso pós-vacinação pode desencadear rumores na comunidade, comprometendo a confiabilidade do Programa Nacional de Imunizaçóes (PNI), com consequências prejudiciais à saúde pública, como a redução da cobertura vacinal e o ressurgimento de doenças imunopreveníveis. ${ }^{(1)}$ Assim, a vigilância e o monitoramento des- ses eventos adversos ou de qualquer outro problema relacionado à vacinação são essenciais, para que os riscos não excedam os benefícios alcançados pelos PNI. ${ }^{(2)}$ A maioria dos países possui sistemas de vigilância, nos quais a notificaçáo da ocorrência de evento adverso pós-vacinação pode ser realizada espontânea e ativamente por qualquer indivíduo, seja ele profissional de saúde ou não. ${ }^{(4,5,7)}$ No Brasil, as notificaçóes são realizadas de forma passiva, pelos profissionais de saúde, quando do retorno do indivíduo que recebeu algum imunobiológico e este lhe causou algum evento adverso pós-vacinação, nos serviços de saúde, ${ }^{(8)}$ levando à subnotificaçáo.

A maioria dos estudos realizados é desenvolvida a partir de dados secundários do Sistema de Informação dos Eventos Adversos Pós-Vacinação (SI-EAPV) brasileiro. Entretanto, os problemas no preenchimento dos formulários de notificação, principalmente no que diz respeito à completude dos campos, pode interferir na qualidade das informaçóes e, consequentemente, na real situação da ocorrência do evento. ${ }^{(9-11)}$

A proposta deste estudo avança no conhecimento ao propor realizar vigilância ativa dos eventos adversos pós-vacinação, com uma busca direta de dados, permitindo maior conhecimento desses eventos na comunidade. Esse tipo de vigilância permite observar, conhecer e identificar evento adverso 
pós-vacinação, planejar ações de saúde e melhorar a comunicação entre profissionais de saúde e usuários, possibilitando aumentar a confiança dos indivíduos em relação aos imunobiológicos disponíveis nos PNI. ${ }^{(12)}$

No Brasil, a equipe de enfermagem ocupa posição de destaque, no que se refere às atividades de vacinação. A enfermagem é responsável por todas as etapas do processo de vacinação no nível local de saúde, representado pelas salas de vacinação, desde o acolhimento do usuário; conservação e administração dos imunobiológicos; manuseio do Sistema de Informação do Programa Nacional de Imunizações (SI-PNI), além da vigilância dos eventos adversos.

O presente estudo objetivou descrever a vigilância ativa dos eventos adversos pós-vacinação, sua incidência e os fatores associados, em um município de Minas Gerais, Brasil. A maioria dos estudos realizados no Brasil baseia-se no banco de dados disponibilizado pelo SI-EAPV; ${ }^{(11)}$ estudos epidemiológicos de coorte são essenciais para complementar tais informaçóes, a fim de identificar a incidência e os fatores associados aos eventos adversos pós-vacinação; contribuir para a segurança nas salas de vacinação e, consequentemente, manter a confiabilidade dos PNI.

\section{Métodos}

Trata-se de estudo epidemiológico observacional de coorte prospectiva, realizado na Atenção Primária à Saúde (APS) de um município de Minas Gerais, na Região Sudeste do Brasil.

O cenário desta pesquisa foi composto de todas as 43 unidades de APS do município, distribuídas em dez regióes sanitárias. Para este estudo, foi realizado o agrupamento dessas regióes, considerando a população e a proximidade territorial, resultando em seis regióes sanitárias. Posteriormente, foram selecionadas, por amostra de conglomerado simples, seis unidades de APS, estratificadas pelas seis regióes sanitárias. As unidades foram selecionadas considerando sua localização estratégica e por apresentarem grande fluxo de atendimentos, o que acabou por favorecer a oportunidade de encontrar indivíduos disponíveis para o estudo. Os indivíduos foram distribuídos proporcionalmente ao tamanho de cada região sanitária.

A amostra da coorte foi calculada considerando a populaçáo cadastrada e atendida no sistema público de saúde municipal $(\mathrm{n}=187.030)$. Utilizou-se a estimativa de proporção de $50 \%$ para determinada característica, valor este que fornece o maior tamanho amostral para população finita, fixando o nível de significância em 5\% e o erro amostral em 5\%. A amostra estimada para a composição da coorte foi de 384 indivíduos.

Foram incluídos no estudo indivíduos que compareceram às unidades de APS para receber algum tipo de vacina ofertada pelo PNI brasileiro e tivessem telefone, para faciliar seu acompanhamento. Foi garantida a participação de indivíduos público-alvos dos calendários de vacinação contemplados no PNI brasileiro. Excluíram-se os indivíduos de qualquer idade que compareceram para receber vacinas especiais em decorrência de evento adverso prévio à vacinação.

A coleta de dados ocorreu entre setembro de 2017 e junho de 2018. A pesquisa de campo prolongou-se em decorrência do tamanho da amostra, da logística e das recusas $(n=189)$ por parte da população em participar da pesquisa. Durante essa etapa, seis pesquisadores, previamente treinados, realizaram a coleta dos dados na linha de base, trabalhando em escala de revezamento nas unidades selecionadas, por um período de 4 a 5 horas diárias. A coleta foi realizada em uma unidade de saúde por vez. $\mathrm{O}$ inquérito telefônico foi realizado somente pela pesquisadora principal.

Utilizou-se um questionário semiestruturado, adaptado do formulário de notificação/investigação de eventos adversos pós-vacinação, padronizado pelo Sistema Nacional de Vigilância Epidemiológica. ${ }^{(2)}$ O questionário foi composto por um conjunto de itens relacionados a: identificação sociodemográfica, informaçôes de saúde e antecedentes vacinais, informaçóes sobre vacinas e eventos adversos pós-vacinação e orientaçóes sobre a vacinação.

$\mathrm{Na}$ linha de base, os indivíduos que compareceram às unidades de APS para vacinação e concordaram em participar do estudo foram entrevistados 
individualmente. A entrevista foi realizada em uma sala privativa, na própria Unidade Básica de Saúde, após a administração da vacina, e teve duração média de 15 minutos. Após a entrevista, os participantes foram orientados quanto ao seguimento do acompanhamento, a ser realizado por contato telefônico após 72 horas da vacinação. A intervenção telefônica é uma estratégia contemporânea, acessível e de baixo custo para acompanhamento individual, que pode aumentar o vínculo e o acesso aos serviços de saúde. ${ }^{(13)}$ A decisão pelo período de acompanhamento de 72 horas se deveu à maior prevalência de eventos adversos pós-vacinação nesse período. ${ }^{(2)}$

No seguimento, o indivíduo foi novamente entrevistado quanto à presença ou não de eventos adversos pós-vacinação e, mediante relato do evento, procedeu-se a orientaçóes quanto aos cuidados e à notificação do evento. Para indivíduos que não foram encontrados nas 72 horas de seguimento, novos contatos telefônicos foram feitos, na tentativa de reduzir a perda amostral. No período do seguimento, realizou-se também a busca no SI-EAPV municipal, a fim de identificar os eventos notificados e confirmados nas unidades de APS selecionadas.

A variável desfecho foi a presença de eventos adversos pós-vacinação com início dos sintomas até 72 horas. Os critérios de definição de eventos adversos pós-vacinação foram: relatos de manifestaçôes locais (dor, vermelhidão, endurecimento e edema) e manifestaçóes sistêmicas (febre termométrica, choro persistente, dor de cabeça, vômitos, diarreia, episódio hipotônico hiporresponsivo. Para o episódio hipotônico hiporresponsivo foram levados em consideração, no relato do indivíduo, sintomas que identificassem hipotonia, hiporresponsividade e cianose. Para o choro persistente, considerou-se o relato de choro prolongado e inconsolável por mais de 6 horas. ${ }^{(2)}$ A vacina composta pelo bacilo de CalmetteGuérin (BCG), por apresentar normalmente evento adverso após 72 horas, foi excluída do estudo.

As variáveis de exposição foram: identificação sociodemográfica (sexo, idade, data de nascimento, endereço e contato telefônico); informações sobre saúde e histórico vacinal (doenças preexistentes, alergias conhecidas, medicamentos em uso, sintomas clínicos no momento da vacinação e presença de evento adverso pós-vacinação em doses anteriores); vacinação atual (data da vacinação, unidade de APS, categoria profissional do administrador e quantidade de vacinas administradas); características do evento adverso pós-vacinação e as ações de vigilância epidemiológica (data de identificação e tipo de evento, necessidade de encaminhamento a outros serviços de saúde, conduta do profissional de saúde, hospitalização, presença de manifestaçôes sistêmicas e recebimento de informaçóes acerca das diretrizes de vacinação e eventos adversos pós-vacinação).

Para a análise e tratamento dos dados, foi utilizado o software Stata (versão 14.0). Calcularam-se a distribuição de frequências relativas para as variáveis categóricas e mediana para a variável idade. A incidência de evento adverso pós-vacinação foi estimada considerando os casos de eventos como numerador e o número de doses de vacinas aplicadas no período do estudo no denominador. ${ }^{(2)}$ Foi utilizado o SI-PNI municipal para o levantamento das doses de vacinas aplicadas no período do estudo.

Para a análise bivariada, foi utilizado o teste do qui-quadrado de Pearson. As variáveis explicativas, que obtiveram valor de $\mathrm{p}$ menor que $20 \%(\mathrm{p}<0,20)$, foram inseridas pelo método backward no modelo multivariado de regressão de Poisson de variância robusta, para verificar os fatores associados aos eventos adversos pós-vacinação. Aqueles com menor significado (valor mais alto de $\mathrm{p}$ ) foram removidos um a um do modelo. O procedimento foi repetido até que todas as variáveis presentes no modelo apresentassem significância estatística, com p<0,05. Cabe ressaltar que o modelo multivariado foi controlado pelo efeito da variável idade dos indivíduos, categorizada em: menor de 1 ano; 1 a 4 anos; 5 a 19 anos e 20 anos ou mais. Nessa análise, agruparam-se em uma única categoria adultos e idosos, devido à baixa ocorrência de evento adverso pós-vacinação observada nessas faixas etárias. O teste de HosmerLemeshow foi utilizado para verificar o ajuste do modelo final. $\mathrm{O}$ risco relativo (RR), com intervalo de confiança de 95\% (IC95\%), foi usado como medida de efeito. Para todas as análises, foi utilizado o nível de significância de 5\%.

O estudo foi aprovado pelo Comitê de Ética em Pesquisa, sob o parecer 2.206.213. 


\section{Resultados}

Do total de indivíduos vacinados entrevistados $(\mathrm{n}=384)$, mais da metade eram do sexo feminino e branca, com idades entre zero e 83 anos, com mediana de 28,5 anos. Em relação à faixa etária, 32,3\% eram crianças menores de 5 anos. Na amostra, identificaram-se 2,6\% de gestantes, mas nenhuma delas apresentou evento adverso pós-vacinação.

Quanto ao histórico de informaçôes e saúde, mais da metade afirmou não ter nenhum tipo de comorbidade. Entre as comorbidades autorrelatadas, as mais citadas foram: doenças cardíacas $(18,5 \%)$ e diabetes $(6,8 \%)$; consequentemente, os usos de anti-hipertensivos $(14,3 \%)$ e de antidiabético $(4,7 \%)$ foram os mais citados. Além disso, 2,9\% dos indivíduos relataram já ter tido pelo menos um episódio convulsivo, $8,4 \%$ relataram alergia a medicamentos e $1,8 \%$ alergia à lactose.

A maioria das vacinas foi aplicada pelo profissional técnico de enfermagem $(97,4 \%)$. Dentre os vacinados, $78,1 \%$ relataram que nenhuma triagem vacinal foi realizada; mais da metade não recebeu orientaçóes acerca da vacina administrada, não foi informada sobre a possibilidade de evento adverso e não recebeu orientação sobre a conduta caso um evento desse tipo ocorresse.

Entre os indivíduos, 62,2\% receberam apenas uma vacina, enquanto os demais variaram de duas a três ou mais. A via de administraçáo mais utilizada foi a intramuscular.

No seguimento, houve perda amostral de 7,5\% $(\mathrm{n}=29$, devido à impossibilidade de contato com o indivíduo vacinado, conforme número ou endereço de telefone informados. Entre os 355 indivíduos vacinados acompanhados, 35,8\% ( $\mathrm{n}=127)$ autodeclararam presença de algum tipo de evento adverso pós-vacinação.

A tabela 1 apresenta as características clínicas e epidemiológicas dos indivíduos vacinados que relataram presença de evento adverso pós-vacinaçáo $(\mathrm{n}=127)$. Os eventos locais foram os mais relatados e incluíram dor, endurecimento e vermelhidáo no local de administração da vacina. Em relaçáo aos eventos sistêmicos, os indivíduos vacinados relataram presença de febre $\geq 37,5^{\circ} \mathrm{C}$, cefaleia e choro persistente.
Outros sintomas, como diarreia, episódio hipotônico hiporresponsivo, náusea e vômitos, também foram citados. Ressalta-se que, na análise, identificou-se mais de um sintoma por indivíduo vacinado. Em relaçáo ao tempo de início do evento adverso, 20,5\% ocorreram menos de 1 hora após a administraçáo da vacina; $40,2 \%$ em mais de 1 hora, e $39,3 \%$ em mais de 12 horas, náo excedendo o tempo de seguimento de 72 horas. Dos 127 indivíduos vacinados que relataram a presença de evento adverso pós-vacinação, $26,0 \%$ procuraram serviços de saúde, incluindo as salas de vacinação das unidades de APS e hospitais. Apenas 17,3\% foram notificados e investigados e, destes, 4,7\% foram hospitalizados. A maioria dos eventos adversos pós-vacinação relatados foi classificada como năo grave $(81,9 \%)$.

Tabela 1. Características clínicas e epidemiológicas de indivíduos vacinados que relataram presença de eventos adversos pós-vacinação

\begin{tabular}{|c|c|}
\hline Variáveis clínicas e epidemiológicas & $\mathrm{n}(\%)$ \\
\hline \multicolumn{2}{|l|}{ Tipo de reações relatadas } \\
\hline Eventos locais & $71(55,9)$ \\
\hline Eventos locais e sistêmicos simultâneos & $39(30,7)$ \\
\hline Eventos sistêmicos & $17(13,4)$ \\
\hline \multicolumn{2}{|l|}{ Reações locais } \\
\hline Dor local e vermelhidão & $120(31,2)$ \\
\hline Edema e endurecimento & $36(9,3)$ \\
\hline \multicolumn{2}{|l|}{ Reações sistêmicas } \\
\hline Febre $\geq 37,5^{\circ} \mathrm{C}$ & $23(6,0)$ \\
\hline Cefaleia, náuseas e diarreia & $17(4,3)$ \\
\hline Choro persistente e EHH & $8(2,1)$ \\
\hline \multicolumn{2}{|l|}{ Tempo de início dos sintomas } \\
\hline $1-12$ horas & $51(40,2)$ \\
\hline $12-72$ horas & $50(39,3)$ \\
\hline 15 minutos a 1 hora & $18(14,2)$ \\
\hline$<15$ minutos & $8(6,3)$ \\
\hline Nenhum & $89(70,1)$ \\
\hline Sala de vacinação das unidades de APS & $20(15,8)$ \\
\hline Hospital & $7(5,5)$ \\
\hline Consultório médico privado & $6(4,7)$ \\
\hline \multicolumn{2}{|l|}{ Até 72 horas de internação } \\
\hline Não & $121(95,3)$ \\
\hline $\operatorname{Sim}$ & $6(4,7)$ \\
\hline \multicolumn{2}{|l|}{ EAPV notificado e investigado } \\
\hline Não & $105(82,6)$ \\
\hline Sim & $22(17,3)$ \\
\hline \multicolumn{2}{|l|}{ Classificação do EAPV confirmado } \\
\hline EAPV não grave & $18(81,9)$ \\
\hline EAPV grave & $4(18,1)$ \\
\hline
\end{tabular}
à Saúde

$\mathrm{Na}$ busca no SI-EAPV no período do estudo, foram identificados oito erros de vacinação notifica- 
dos pelas unidades de APS participantes do estudo. No entanto, esses erros não causaram eventos adversos e também não fizeram parte do estudo. Além disso, seis eventos adversos pós-vacinação relacionados à vacina BCG foram identificados, mas não entraram na análise do estudo porque tiveram início após 72 horas.

Para o cálculo da incidência, foram analisados apenas os casos de eventos adversos pós-vacinação confirmados $(\mathrm{n}=22)$, considerando 164.640 doses de vacinas aplicadas no período estudado, nas regióes selecionadas. A incidência foi de 13,36 casos/100 mil doses de vacinas aplicadas (IC95\% 13,34-13,38). A maior incidência foi em crianças menores de 5 anos $(45,6 \%)$, e os eventos mais frequentes foram dor, vermelhidão e endurecimento, seguidos de febre, edema, episódio hipotônico hiporresponsivo, diarreia e choro persistente.

$\mathrm{Na}$ análise bivariada, a incidência de eventos adversos pós-vacinação foi maior entre os indivíduos vacinados que receberam orientaçóes sobre as vacinas e os ocasionados por elas e sobre a conduta a ser tomada diante da presença desses eventos. As vias de administração intramuscular e oral também estiveram associadas à presença de eventos adversos pós-vacinação (Tabela 2).

Em relação à distribuiçãa percentual de eventos adversos pós-vacinação, de acordo com as vacinas recebidas no momento da coleta de dados, identificou-se que as vacinas contra hepatite $\mathrm{B}$ e influenza estavam associadas a um risco maior de evento adverso (Tabela 3).

$\mathrm{Na}$ análise multivariada, quando ajustada pela idade, observou-se que o recebimento de orientaçóes sobre as vacinas administradas aumentou 3,4 vezes a notificação de eventos adversos pós-vacinação. A vacina administrada por via intramuscular aumentou o risco de eventos adversos em até 6,1 vezes. Quanto às vacinas recebidas, a vacina contra difteria e tétano aumentou o risco de ter evento adverso pós-vacinação em até 7,9 vezes, enquanto a vacina contra hepatite $B$ reduziu esse risco. As demais variáveis não apresentaram significância estatística, sendo excluídas do modelo ajustado, pois apresentaram valor de p> 0,05 (Tabela 4).
Tabela 2. Eventos adversos pós-vacinação confirmados, de acordo com características sociodemográficas e histórico de saúde de indivíduos vacinados, atividades realizadas em serviços de imunização e vacinas aplicadas

\begin{tabular}{|c|c|c|c|c|}
\hline \multirow{2}{*}{ Variáveis } & \multirow{2}{*}{$\begin{array}{l}\text { Total } \\
\mathrm{n}(\%)\end{array}$} & \multicolumn{2}{|c|}{ EAPV (\%) } & \multirow{2}{*}{$p$-value } \\
\hline & & Sim & Não & \\
\hline \multicolumn{5}{|l|}{ Sexo } \\
\hline Feminino & $209(54,4)$ & 6,7 & 93,3 & \\
\hline Masculino & $175(45,6)$ & 4,5 & 95,5 & 0,372 \\
\hline \multicolumn{5}{|c|}{ Grupo de idade, anos } \\
\hline$\geq 20$ & $242(57,0)$ & 5,0 & 95,0 & \\
\hline $1-4$ & $54(19,8)$ & 6,6 & 93,4 & 0,915 \\
\hline$<1$ & $48(12,5)$ & 6,3 & 93,7 & \\
\hline 5-19 & $40(10,7)$ & 7,3 & 92,7 & \\
\hline \multicolumn{5}{|l|}{ Etnia } \\
\hline Branco & $202(52,6)$ & 6,4 & 93,6 & 0,530 \\
\hline Não branco & $182(47,4)$ & 4,9 & 95,1 & \\
\hline \multicolumn{5}{|l|}{ Gestante } \\
\hline Não & $374(97,4)$ & 5,8 & 94,2 & \\
\hline Sim & $10(2,6)$ & - & 100,0 & 0,430 \\
\hline \multicolumn{5}{|l|}{ Infantil } \\
\hline Não & $323(84,1)$ & 5,2 & 94,8 & \\
\hline $\operatorname{Sim}$ & $61(15,9)$ & 8,2 & 91,8 & 0,366 \\
\hline \multicolumn{5}{|c|}{ Orientação sobre a vacina } \\
\hline Não & $236(61,5)$ & 3,4 & 96,6 & \\
\hline $\operatorname{Sim}$ & $148(38,5)$ & 9,4 & 90,6 & 0,013 \\
\hline \multicolumn{5}{|c|}{ Informações específicas sobre EAPV } \\
\hline Não & $228(59,4)$ & 3,5 & 96,5 & \\
\hline $\operatorname{Sim}$ & $156(40,6)$ & 9,0 & 91,0 & 0,024 \\
\hline \multicolumn{5}{|c|}{ Conduta em caso de EAPV } \\
\hline Não & $267(69,5)$ & 3,7 & 96,3 & \\
\hline $\operatorname{Sim}$ & $117(30,5)$ & 10,6 & 89,8 & 0,012 \\
\hline \multicolumn{5}{|c|}{ Número de vacinas recebidas } \\
\hline Uma & $239(62,2)$ & 6,3 & 93,7 & \\
\hline Três ou mais & $74(19,3)$ & 4,0 & 96,0 & \\
\hline Duas & $71(18,5)$ & 5,6 & 94,4 & 0,772 \\
\hline \multicolumn{5}{|l|}{ Via intramuscular } \\
\hline Não & $337(87,8)$ & 4,4 & 95,6 & 0,004 \\
\hline Sim & $47(12,2)$ & 14,9 & 85,1 & \\
\hline \multicolumn{5}{|l|}{ Via subcutânea } \\
\hline Sim & $290(75,5)$ & 9,5 & 90,5 & \\
\hline Não & $94(24,5)$ & 4,5 & 95,5 & 0,065 \\
\hline \multicolumn{5}{|l|}{ Via oral } \\
\hline Sim & $347(90,4)$ & 16,2 & 83,8 & \\
\hline Não & $37(9,6)$ & 4,6 & 95,4 & 0,004 \\
\hline
\end{tabular}

'Teste do qui-quadrado de Pearson. EAPV - Evento Adverso Pós-Vacinação

\section{Discussão}

Embora a vigilância passiva consiga manter e alimentar um sistema de informação e seja um serviço de baixo custo, ela tem a desvantagem de subnotificar os eventos adversos pós-vacinaçãao ${ }^{(9,12,14)}$ e reduzir a sensibilidade para a identificação de novos casos, sinais ou mesmo novos tipos de eventos adversos. ${ }^{(15)}$

Estudo observacional, realizado na região de Puglia, na Itália, mostrou que os serviços de saúde 
Tabela 3. Eventos adversos pós-vacinação, de acordo com as vacinas recebidas

\begin{tabular}{|c|c|c|c|c|}
\hline \multirow{2}{*}{ Vacinas } & \multirow{2}{*}{ Total (\%) } & \multicolumn{2}{|c|}{ EAPV (\%) } & \multirow{2}{*}{$p$-value ${ }^{*}$} \\
\hline & & Sim & Não & \\
\hline \multicolumn{5}{|c|}{ Hepatite B } \\
\hline Não & 71,4 & 7,3 & 92,7 & \\
\hline Sim & 28,6 & 1,8 & 98,2 & 0,037 \\
\hline \multicolumn{5}{|l|}{ dT } \\
\hline Não & 76,3 & 4,8 & 95,2 & \\
\hline $\operatorname{Sim}$ & 23,7 & 8,8 & 91,2 & 0,150 \\
\hline \multicolumn{5}{|l|}{ Penta } \\
\hline Não & 93,2 & 5,8 & 94,2 & \\
\hline Sim & 6,8 & 3,8 & 96,2 & 0,669 \\
\hline \multicolumn{5}{|l|}{ FLU3V } \\
\hline Não & 70,3 & 7,4 & 92,6 & \\
\hline Sim & 29,7 & 1,8 & 98,2 & 0,029 \\
\hline \multicolumn{5}{|l|}{ SCR } \\
\hline Não & 88,5 & 5,0 & 95,0 & \\
\hline Sim & 11,5 & 11,4 & 88,6 & 0,087 \\
\hline \multicolumn{5}{|c|}{ Febre amarela } \\
\hline Não & 89,3 & 5,5 & 84,5 & \\
\hline Sim & 10,7 & 7,3 & 92,7 & 0,643 \\
\hline \multicolumn{5}{|l|}{ VIP } \\
\hline Não & 91,9 & 5,9 & 94,1 & \\
\hline Sim & 8,1 & 3,2 & 96,8 & 0,532 \\
\hline \multicolumn{5}{|c|}{ Pneumo 10} \\
\hline Não & 91,1 & 6 & 94 & \\
\hline Sim & 8,9 & 2,9 & 97,1 & 0,464 \\
\hline \multicolumn{5}{|c|}{ Meningo C } \\
\hline Não & 91,9 & 6,3 & 93,7 & \\
\hline Sim & 8,1 & - & 100 & 0,152 \\
\hline \multicolumn{5}{|l|}{ DTP } \\
\hline Não & 95,8 & 5,4 & 94,6 & \\
\hline Sim & 4,2 & 12,5 & 87,5 & 0,234 \\
\hline \multicolumn{5}{|l|}{ Varc } \\
\hline Não & 95,6 & 5,7 & 94,3 & \\
\hline Sim & 4,4 & 5,9 & 94,1 & 0,978 \\
\hline \multicolumn{5}{|l|}{ HPV } \\
\hline Não & 96,6 & 5,6 & 94,4 & \\
\hline Sim & 3,4 & 7,7 & 92,3 & 0,757 \\
\hline \multicolumn{5}{|l|}{ VOP } \\
\hline Não & 96,1 & 5,4 & 95,6 & \\
\hline Sim & 3,9 & 13,3 & 86,7 & 0,196 \\
\hline \multicolumn{5}{|l|}{ ROTA } \\
\hline Não & 94,8 & 5,2 & 94,8 & \\
\hline Sim & 5,2 & 15 & 85 & 0,067 \\
\hline \multicolumn{5}{|c|}{ Hepatite A } \\
\hline Não & 98,7 & 5,4 & 94,6 & \\
\hline Sim & 1,3 & 20 & 80 & 0,167 \\
\hline \multicolumn{5}{|l|}{ SCRV } \\
\hline Não & 99,5 & 5,8 & 842 & \\
\hline Sim & 0,5 & - & 100 & 0,727 \\
\hline dTpa & & & & \\
\hline Não & 98,4 & 5,8 & 94,2 & \\
\hline Sim & 1,6 & - & 100 & 0,543 \\
\hline
\end{tabular}

'Teste qui-quadrado de Pearson. EAPV - Evento Adverso Pós-Vacinação; dT - Vacina contra difteria e tétano (formulação para adultos/adolescentes); Penta - vacina pentavalente (contra difteria + tétano + coqueluche + Haemophilus influenzae tipo B + hepatite B); FLU3V - Vacina contra gripe trivalente inativada e fragmentada; SCR - Vacina contra sarampo + caxumba + rubéola; VIP - Vacina Inativada contra a Poliomielite; Pneumo 10 - Vacina pneumocócica 10-valente; meningo $\mathrm{C}$ - vacina meningocócica do tipo C; DTP - Vacina Contra Difteria + Tétano + Coqueluche adsorvida; Varc - Vacina contra varicela; HPV: Vacina quadrivalente contra o papilomavírus humano do tipos 6,11,16 e 18 recombinante; VOP - Vacina atenuada Oral contra a Poliomielite; ROTA - Vacina contra o rotavírus humano G1P1 [8]; SCRVVacina contra sarampo + caxumba + rubéola + varicela; dTpa - Vacina acelular contra difteria + tétano + coqueluche
Tabela 4. Modelo final de regressão de Poisson para fatores associados aos eventos adversos pós-vacinação

\begin{tabular}{|c|c|c|c|}
\hline Variáveis & RR & IC95\%* & $p$-value ${ }^{t}$ \\
\hline \multicolumn{4}{|c|}{ Orientação sobre as vacinas } \\
\hline Sim & 3,4 & $1,53-7,68$ & 0,003 \\
\hline Não & 1 & - & \\
\hline \multicolumn{4}{|c|}{ Via intramuscular } \\
\hline Sim & 6,1 & $2,55-14,63$ & $<0,001$ \\
\hline Não & 1 & - & \\
\hline \multicolumn{4}{|c|}{ Vacina contra hepatite B } \\
\hline Sim & 0,1 & $0,03-0,85$ & 0,031 \\
\hline Não & 1 & - & \\
\hline \multicolumn{4}{|l|}{ Vacina dT } \\
\hline Sim & 7,9 & $2,77-22,46$ & $<0,001$ \\
\hline Não & 1 & - & \\
\hline
\end{tabular}

que realizam busca e vigilância ativa de eventos adversos pós-vacinação aumentam consideravelmente o número dessas notificaçóes. ${ }^{(16)}$ Isso comprova os resultados evidenciados neste estudo em relação aos eventos adversos relatados pelos indivíduos e aos relatados nas unidades de APS. Mesmo na ocorrência de eventos adversos pós-vacinação, a maioria dos indivíduos vacinados não retornou às salas de vacinação das unidades de APS para a devida notificação.

Os resultados deste estudo demonstram incidência de eventos adversos pós-vacinação consistente com a descrita na literatura, ${ }^{(5,6,9)}$ em que crianças menores de 5 anos apresentaram maior probabilidade de desenvolver esse tipo de evento. A alta incidência pode estar associada à imaturidade do sistema imunológico e ao alto número de vacinas administradas nessa faixa etária. ${ }^{(17-20)}$

Entre os eventos locais e sistêmicos identificados, a dor e a febre foram os mais incidentes. A maioria dos eventos adversos pós-vacinação, locais e sistêmicos, são leves e autolimitantes ${ }^{(8,9,17,21)}$ e representam a área de atuação da equipe de enfermagem, uma vez que, nas unidades de APS públicas brasileiras, ela é a principal responsável pelas atividades de vacinação. ${ }^{(14)} \mathrm{O}$ fato de as reaçôes locais serem poucas e intensas, além de esperadas pelo indivíduo vacinado, acabam náo sendo relatadas às unidades de APS. ${ }^{(5,8)}$

Nesse aspecto, é importante enfatizar a atuação da equipe de enfermagem na vigilância de eventos adversos pós-vacinação. Os achados deste estudo identificaram que o fato de o profissional fazer orien- 
taçóes sobre a vacinação aumenta a notificação voluntária desse tipo de evento e, consequentemente, sua incidência. No entanto, mais da metade dos indivíduos vacinados relatou que não tinha recebido informaçóes básicas sobre as vacinas administradas, quais doenças estavam prevenindo e que possíveis eventos adversos poderiam ser causados por elas. Profissionais de saúde com conhecimento são capazes de informar os indivíduos sobre a importância e os benefícios da vacinação e sobre os possíveis riscos e a ocorrência de eventos adversos pós-vacinação. ${ }^{(16,20)} \mathrm{O}$ fornecimento dessas informaçóes à população, chamado de triagem vacinal, é recomendado como atividade básica a ser realizada nas salas de vacinação. ${ }^{(10)} \mathrm{A}$ triagem vacinal é uma medida específica para evitar riscos na vacinação, pois, além de permitir o monitoramento do status vacinal dos indivíduos e de seus históricos de saúde, é quando o profissional de saúde tem a oportunidade de realizar aconselhamentos sobre as vacinas e seus possíveis EAPV. ${ }^{(14)}$

Quando não há esclarecimentos sobre os benefícios da vacinação e possíveis eventos adversos pós-vacinação, a população tende a se retirar dos serviços de saúde, comprometendo as próximas doses, tanto por medo de outras reaçóes quanto por insegurança no profissional vacinador, ${ }^{(9)}$ aumentando o risco de adquirir doenças imunopreveníveis. Com isso, tais doenças, que já foram controladas, podem ressurgir. ${ }^{(8)}$ Os estudos enfatizam que a triagem vacinal adequada e a capacitação e a educação em saúde são medidas específicas para aumentar a notificação dos eventos adversos pós-vacinação e garantir a qualidade e a segurança da vacinação. ${ }^{(17)}$ Além disso, o conhecimento sobre vacinas e seus possíveis eventos adversos aumenta consideravelmente a confiança da população em relação à vacinação, uma vez que os usuários se sentem bem-vindos nas salas de vacinação, e isso lhes permite dirimir as dúvidas existentes.

A vacina contra difteria e tétano foi associada a um risco aumentado de causar evento adverso. Essa vacina agrega os toxóides tetânico e difteria, bem como o hidróxido de alumínio e o timerosal, considerado um dos principais responsáveis por causar reaçôes locais. (22) A falta de conhecimento do histórico vacinal em adultos, devido à falta de evidências da administração das vacinas, favorece o desenvolvimento de eventos adversos pós-vacinação, devido à revacinação em um período menor do que o recomendado. ${ }^{(6,17)}$

Outro achado do estudo refere-se à menor reatogenicidade da vacina contra hepatite B. Estudos brasileiros e internacionais comprovam a eficácia e a segurança dessa vacina. ${ }^{(1,23)}$ É importante ressaltar que as vacinas administradas durante este estudo, em geral, tiveram a mesma origem de fabricação, sendo grande parte produzida em laboratórios brasileiros, o que demonstra a qualidade dos imunobiológicos produzidos nacionalmente.

Não houve associação significativa entre o número de vacinas recebidas e a presença de eventos adversos pós-vacinação. Estudo de revisão sistemática indicou a ausência de eventos adversos pós-vacinação na presença de administração de três ou mais doses de vacinas. ${ }^{(24)}$ Por outro lado, a via de administração intramuscular foi associada à presença desse tipo de evento. Isso se deve ao fato de a maioria das vacinas, por serem inativadas, exigir adjuvantes, geralmente derivados do alumínio, o que aumenta o risco de reaçóes locais. ${ }^{(15)}$

Nesse sentido, o profissional de saúde precisa ter conhecimentos teóricos e práticos para a administração de vacinas pela via intramuscular, para não incorrer em erros que possam gerar eventos adversos pós-vacinação e insatisfação do indivíduo vacinado. ${ }^{(14,25)}$ Certas precauçôes devem ser tomadas ao administrar uma vacina por via intramuscular, considerando a composição corporal do indivíduo vacinado, o tamanho da agulha e o volume a ser administrado. ${ }^{(2)}$

A qualidade metodológica deste estudo forneceu dados baseados em evidências para estimar a incidência e o risco de eventos adversos pós-vacinação entre indivíduos vacinados. $\mathrm{O}$ estudo de coorte oferece melhores estimativas de associação e permite uma cronologia precisa entre as exposiçóes e o evento. No entanto, deve-se considerar que a existência de nexo causal entre o evento e os possíveis fatores de exposição é complexa e pressupõe análise cuidadosa da qualidade e da consistência dos dados. Os estudos de coorte sobre eventos adversos pós-vacinação, após o licenciamento de vacinas, ainda são escassos, com a maioria baseando-se em registros dos sistemas de informação em imunização. ${ }^{(8,9)}$ 
Com os resultados deste estudo, avança-se no conhecimento, ao evidenciar, por meio de testes de análise multivariada, a importância da conduta dos profissionais de enfermagem na orientação em sala de vacinação, para aumentar a notificaçáo e a investigação de eventos adversos pós-vacinação e, consequentemente, o fortalecimento da vigilância ativa deles.

Como limitação deste estudo, a inclusão de todas as faixas etárias na identificação de eventos adversos pós-vacinação dificultou a comparação dos resultados com a literatura científica, uma vez que grande parte das pesquisas sobre esse tema é realizada em crianças. Outro fator limitante é o curto período de acompanhamento após a vacinação (72 horas), o que pode ter contribuído para a não observação de outros possíveis eventos que ocorreram no período superior de horas em comparação ao estabelecido para essa coorte.

\section{Conclusão}

O estudo reforça a importância da vigilância ativa dos eventos adversos pós-vacinação no Brasil e aponta para deficiências na vigilância passiva, nas salas de vacinação das unidades de Atenção Primária à Saúde, uma vez que esta não retrata todos os casos de eventos desse tipo que ocorreram. Os resultados evidenciados contribuem para destacar a subnotificação de eventos adversos pós-vacinação e a importância da conduta dos profissionais de enfermagem em relação às orientações em vacinação. Tais achados podem subsidiar a implementação de boas práticas nas salas de vacinação das unidades de Atenção Primária à Saúde e ser úteis para futuros estudos epidemiológicos, relacionados a erros de imunização identificados, mas não explorados neste estudo.

\section{Agradecimentos}

À Fundação de Amparo à Pesquisa do Estado de Minas Gerais (FAPEMIG) e Ministério da Saúde - Programa de Pesquisa do SUS (PPSUS - APQ-03509-13). À Coordenação de Aperfeiçoamento de Pessoal de Nível Superior (CAPES - Código de Financiamento 001).

\section{Colaborações}

Batista ECC, Ferreira AP, Oliveira VC, Amaral GG, Jesus RF, Quintino ND, Viegas SMF e Guimarães EAA contribuíram com a concepção do estudo, análise e interpretação dos dados, redação do artigo, revisão crítica relevante do conteúdo intelectual e aprovação da versão final a ser publicada.

\section{Referências}

1. Di Pasquale A, Bonanni P, Garçon N, Stanberry LR, El-Hodhod M, Tavares Da Silva F. Vaccine safety evaluation: Practical aspects in assessing benefits and risks. Vaccine. 2016;34(52):6672-80. Review.

2. Brasil. Ministério da Saúde. Secretaria de Vigilância em Saúde. Departamento de Vigilância das Doenças Transmissíveis. Manual de vigilância epidemiológica de eventos adversos pós-vacinação. 3a ed. Brasília (DF): Ministério da Saúde; 2014 [citado 2020 Dez 30]. Disponível em: https://bvsms.saude.gov.br/bvs/publicacoes/ manual_vigilancia_epidemiologica_eventos_adversos_pos_ vacinacao.pdf

3. Santos SS, Oliveira VC, Ribeiro HC, Alves TG, Cavalcante RB, Guimarães EA. Analysis of adverse events following immunization in Minas Gerais, Brazil, 2011: a cross-sectional study. Epidemiol Serv Saúde. 2016;25(1):45-54.

4. Donà D, Masiero S, Brisotto S, Gottardello L, Lundin R, Borgia E, et al. Special Immunization Service: a 14-year experience in Italy. PLoS One. 2018;13(4):e0195881.

5. Stone CA Jr, Rukasin CRF, Beachkofsky TM, Phillips EJ. Immunemediated adverse reactions to vaccines. $\mathrm{Br} J$ Clin Pharmacol. 2019;85(12):2694-706. Review.

6. Danova J, Kocourkova A, Celko AM. Active surveillance study of adverse events following immunisation of children in the Czech Republic. BMC Public Health. 2017;17(1):167.

7. Haber P, Parashar UD, Haber M, DeStefano F. Intussusception after monovalent rotavirus vaccine-United States, Vaccine Adverse Event Reporting System (VAERS), 2008-2014. Vaccine. 2015;33(38):48737.

8. Sato AP, Ferreira VL, Tauil MC, Rodrigues LC, Barros MB, Martineli E, et al. Use of electronic immunization registry in the surveillance of adverse events following immunization. Rev Saude Publica. 2018;52:4.

9. Santos MC, Pontes Netto VB, Andrade MS. Prevalence and factors associated with the occurrence of adverse events following immunization in children. Acta Paul Enferm. 2016;29(6):626-32.

10. Yamoah P, Bangalee V, Oosthuizen F. Knowledge and perceptions of adverse events following immunization among healthcare professionals in Africa: a case study from Ghana. Vaccines (Basel). 2019;7(1):28.

11. Bisetto LH, Ciosak SI, Cordeiro TL, Boing MS. Adverse events following immunization of the elderly. Cogitare Enferm. 2016;21(4):1-10.

12. Heininger U, Holm K, Caplanusi I, Bailey SR; CIOMS Working Group on Vaccine Safety. Guide to active vaccine safety surveillance: Report of CIOMS working group on vaccine safety - executive summary. Vaccine. 2017;35(32):3917-21 
13. Mantwill S, Fiordelli M, Ludolph R, Schulz PJ. EMPOWER-support of patient empowerment by an intelligent self-management pathway for patients: study protocol. BMC Med Inform Decis Mak. 2015;15:18.

14. Bisetto LH, Ciosak, Sl. Analysis of adverse events following immunization caused by immunization errors. Rev Bras Enferm. 2017;70(1):81-89.

15. Lopes SR, Perin JL, Prass TS, Carvalho SM, Lessa SC, Dórea JG. Adverse Events Following Immunization in Brazil: Age of Child and Vaccine-Associated Risk Analysis Using Logistic Regression. Int J Environ Res Public Health. 2018;15(6):1149.

16. Stefanizzi P, Stella P, Ancona D, Malcangi KN, Bianchi FP, De Nitto S, et al. Adverse Events Following Measles-Mumps-Rubella-Varicella Vaccination and the Case of Seizures: a Post Marketing Active Surveillance in Puglia Italian Region, 2017-2018. Vaccines (Basel). 2019;7(4):140.

17. Loiola HA, Almeida AA, Pereira FM, Sardinha AH. Eventos adversos pós-vacinação ocorridos em crianças no município de São Luís, Maranhão. Rev Pesq Saúde. 2016;17(1):17-22.

18. Mrozek-Budzyn D, Majewska R, Kiełtyka A. Early exposure to thimerosal-containing vaccines and children's cognitive development. A 9-year prospective birth cohort study in Poland. Eur J Pediatr. 2015;174(3):383-91.
19. Salik E, Løvik I, Andersen KE, Bygum A. Persistent Skin Reactions and Aluminium Hypersensitivity Induced by Childhood Vaccines. Acta Derm Venereol. 2016;96(7):967-71.

20. Sebastian J, Gurumurthy P, Ravi MD, Ramesh M. Active surveillance of adverse events following immunization (AEFI): a prospective 3-year vaccine safety study. Ther Adv Vaccines Immunother. 2019;7:2515135519889000.

21. Santos CA, Costa RS, Silva JL, Santos MR, Gomes BL. Conhecimento, atitude e prática dos vacinadores sobre vacinação infantil em TeresinaPI, 2015. Epidemiol Serv Saúde. 2017;26(1):133-40.

22. Trucchi C, Zoppi G. Decennial diphtheria-tetanus adult boosters: are they really necessary? J Prev Med Hyg. 2015;56(1):E44-E8. Review.

23. Myers TR, McNeil MM, Ng CS, Li R, Lewis PW, Cano MV. Adverse events following quadrivalent meningococcal CRM-conjugate vaccine (Menveo®) reported to the Vaccine Adverse Event Reporting system (VAERS), 2010-2015. Vaccine. 2017;35(14):1758-63.

24. Zafack JG, De Serres G, Kiely M, Gariépy MC, Rouleau I, Top KA; Canadian Immunization Research Network. Risk of Recurrence of Adverse Events Following Immunization: a Systematic Review. Pediatrics. 2017;140(3):e20163707. Review.

25. Martins JR, Alexandre BG, Oliveira VC, Viegas SM. Permanent education in the vaccination room: what is the reality? Rev Bras Enferm. 2018;71(Suppl 1):668-76. 